\title{
Localization of targets by strabismic subjects: Contrasting patterns in constant and alternating suppressors
}

\author{
V. A. MANN, A. HEIN, and R. DIAMOND \\ Massachusetts Institute of Technology, Cambridge, Massachusetts 02139
}

\begin{abstract}
Strabismic subjects in whom vision in one eye is constantly suppressed localized targets at different points in space when using each eye. Whether they were esotropes or exotropes determined the relation between the two locations and the angle of their vergence error correlated with the magnitude of the interocular discrepancy. These relationships suggest that constant suppressors utilize information about posture of the dominant eye in reaching toward targets presented to the normally suppressed eye. Neither orthotropes nor strabismics in whom vision in each eye is alternately suppressed showed this anomaly. These results were attributed to differences in the way in which visual space is represented, an outcome of the pattern of use of the eyes during early development.
\end{abstract}

The image of a fixated target normally falls on corresponding areas of the two retinae. In the absence of fusion, input to one eye may be disregarded or suppressed, producing effectively monocular vision (Brenner, Charles, \& Flynn, 1969; Franscetti \& Burian, 1971; Lombrusco, Duffy, \& Robb, 1969; Lehmulke \& Fox, 1975). Transient monocular suppression occurs in persons with normal vision under conditions in which discordant input to the two eyes precludes fusion (retinal rivalry). Visual anomalies such as aniseikonia and anisometropia produce retinal images which are discordant, and constant suppression of vision in one eye is a frequent result. Misalignment of the visual axes (strabismus), if present early in life, is associated with monocular suppression. Either vision in each eye is alternately suppressed or vision in one eye is constantly suppressed.

Among higher mammals, visual control of movement develops during early exposure in light (Riesen, 1958). In the kitten, visual feedback from selfproduced movement is essential to this development (Hein \& Held, 1962; Held \& Hein, 1963). When such feedback is provided to only one eye, that eye alone becomes able to control guided movements (Hein \& Diamond, 1971). If the two eyes are exposed alter-

This research was supported by N.I.H. Grant 5-R01-NF09279 to Dr. A. Hein, by a grant from the Spencer Foundation to Dr. H.-L. Teuber, and by a National Science Foundation Graduate Traineeship to the senior author. We thank Dr. J. Bauer for his assistance in setting up the pointing apparatus. We also thank Dr. J. Rosner and the staff of the Pediatric Clinic of the New England College of Optometry for their assistance in testing. Reprint requests should be sent to: V. A. Mann, Haskins Laboratories, Inc., 270 Crown Street, New Haven, Connecticut 06510 . nately under conditions which provide different opportunities for motor-visual feedback, each eye becomes able to mediate different behaviors (Hein \& Diamond, 1972).

Monocular suppression of vision in the strabismic subject might have consequences similar in some respects to those of monocular occlusion. The human strabismic with alternating suppression might resemble the kitten reared with alternating monocular exposure in light, acquiring control of guided movement separately and independently with each eye. In this case, the guided behaviors mediated by each eye should be equivalent, since exposure conditions for each eye would have been comparable. Constant suppression of vision in one eye, on the other hand, might prevent that eye from mediating behaviors equivalent to those controlled by the preferred or dominant eye.

These possibilities were examined with regard to hand-eye coordination. Persons with normal vision and strabismic subjects performed a task which required pointing to targets presented to monocular view. During pointing, the subject could not see his hand or arm, thus eliminating error feedback.

\section{EXPERIMENT 1}

\section{Subjects}

\section{Method}

Errors of vergence may be either horizontal or vertical. To simplify testing and subsequent analysis, we dealt only with horizontal errors of vergence and with localization of targets in a horizontal plane. In addition, we tested only strabismic subjects exhibiting a comitant vergence error (i.e., subjects in whom magnitude of vergence error does not vary with the direction of gaze). In all cases, strabismus had been observed prior to age 4 and neither surgical correction nor therapeutic patching had been attempted. These clinical procedures might have altered either the 
vergence relations of the eyes or pattern of use of the eyes so that the current status of the subject would not correspond to his or her status in early life.

Subjects who participated in this study included 33 strabismic individuals and 33 with normal binocular vision (orthotropes). Of the strabismics, 20 constantly suppressed vision in one eye (11 esotropes and 9 exotropes) and 13 alternately suppressed vision in each eye (7 esotropes and 6 exotropes). All subjects were between the ages of 6 and 30 . The adults were self-referred from the M.I.T. population, while children were referred by health officials from public schools in the Boston area.

For all observations of visual status, any corrective lenses which the subject normally wore were removed, and no lenses were worn during the experiment itself. The type of vergence error (esotropic or exotropic) and the pattern of use of the eyes were determined by conventional clinical methods (Burian \& von Noorden, 1974; Reinecke \& Miller, 1966). The subjects were instructed to fixate a target located $33 \mathrm{~cm}$ from their eyes. An offset of corneal reflectances revealed presence and type of vergence error; a patch test supplemented this observation and indicated pattern of use. In the patch test, the subject attempts to maintain fixation of the target with one eye while the other eye is alternately occluded and uncovered. Subjects able to maintain fixation with either eye under these conditions were classified as alternating suppressors. Those unable to maintain fixation of a target with the nondominant eye were classified as constant suppressors.

Monocular acuity of each subject was determined with a Snellen chart at a distance of $6.1 \mathrm{~m}$. The range of acuity among constant suppressors was from $20 / 20$ to $20 / 200$ with interocular differences of 5 to 18 Snellen points. Among alternating suppressors, the range of acuity was from $20 / 20$ to $20 / 150$ with interocular differences of 0 to 8 Snellen points. The range of acuity among orthotropes was from $20 / 20$ to $20 / 200$ with interocular differences of 0 to 15 Snellen points. Eliminating optical correction made the range of acuity among the orthotropes and alternators more similar to that among constant suppressors than it would have been with correction.

\begin{abstract}
Apparatus
An apparatus modeled after that described by Held and Gottlieb (1958) was used to assess visually guided reaching. The seated subject looked through an aperture at a first surface mirror positioned $45^{\circ}$ to the horizontal plane. The mirror prevented view of hand or arm while reflecting visual targets as virtual images which appeared to lie on a surface directly in front of the subject. Four green-light-emitting diodes served as targets; each subtended $0.2^{\circ}$ of visual arc. The targets were located $5^{\circ}$ and $20^{\circ}$ to the left and right of a central red-light-emitting diode, which itself subtended $0.5^{\circ}$ of visual arc. The red diode remained constantly visible; the green target lights were illuminated successively according to a fixed semirandom sequence.
\end{abstract}

\section{Procedure}

Using the method described by Bauer, Woods, and Held (1969), a rubber thimble with an electrode embedded in its tip was placed on the index finger of the subject's preferred hand. As each target light appeared, the subject directed his gaze to it and pointed to its apparent position. Contact of the embedded electrode with a specially prepared surface at the optical distance of the targets $(32.5 \mathrm{~cm})$ completed a circuit and caused the horizontal coordinate of the point of contact to be automatically computed.

Twelve practice markings with binocular view of the targets preceded monocular test trials. During monocular testing, each eye was used during alternate blocks of trials; in each block, each of the four targets was presented three times in a fixed semirandom sequence. The other eye was covered with a soft eye patch. At the conclusion of each block of trials, the subject closed his eyes and moved the patch to cover the other eye. The order in which the dominant and nondominant eyes were used was counterbalanced within each group of subjects.

\section{Variability of Markings}

\section{Results}

For each subject, the variance of markings was computed separately for each target as viewed by each eye. Differences in variance were evaluated with a series of $F$ tests. (In cases where differences fail to reach a conventional level of significance, criterion values of $\mathrm{F}$ are indicated for permitting rejection of the null hypothesis at the .05 level. A similar usage is followed for nonsignificant values of $t$.) The four target positions did not differ significantly in variance of markings, and interocular differences in variance were not significantly different from zero (df $=11,11 ; \mathrm{F}<.3$ or $\mathrm{F}>3.0$ ). Therefore, it was legitimate to compute a pooled variance $\left(s_{p}\right)$ for each subject, collapsing across targets and eyes. The mean value of $s_{p}$ was $2.4^{\circ}$, with intersubject differences not significantly greater than zero ( $\mathrm{df}=\mathbf{8 8 , 8 8}$; $F<.7$ or $F>1.4)$. Thus, neither pattern of use of the two eyes (constant suppression, alternating suppression, or binocular use) nor interocular differences in acuity affected the consistency with which a subject pointed to targets viewed by each eye.

\section{Location of Markings}

For each subject, the centroid of markings mediated by each eye was calculated for each target. Interocular differences in centroids were then computed (right eye minus left eye) and subsequently evaluated with a series of $t$ tests. For alternating suppressors and for orthotropes, interocular differences were not significant ( $d f=22 ;|t|>1.7$ ). In contrast, for constant suppressors interocular differences were highly significant $(\mathrm{df}=22 ;|t|>3.2, p<.001)$. Subsequently, interocular differences were averaged across all four targets to yield a single measure of interocular discrepancy (IDC) for each subject. The mean value of IDC for subjects in each group is given in Figure 1. The sign of IDC was positive among esotropes (mean $=4.5^{\circ}$ ) and negative among exotropes (mean $\left.=-6.7^{\circ}\right)$. Thus, the sign of IDC was related to type of vergence error (negative for exotropes, with $\mathrm{df}=9, \mathrm{r}_{\text {sign test }}=0, \mathrm{p}<.01$; positive for esotropes, with $\mathrm{df}=11, \mathrm{r}_{\text {sign test }}=0, \mathrm{p}<.01$ ). For alternating suppressors, on the other hand, the sign of IDC was not related to type of vergence error (for esotropes, $\mathrm{df}=7, \mathrm{r}_{\text {sign test }}=3, \mathrm{p}<.25$; for exotropes, $\mathrm{df}=6, \mathrm{r}_{\text {sign test }}=3, \mathrm{p}<.25$ ).

It was possible to assess the stability of $s_{p}$ and IDC in some cases. Four of the subjects who participated in this experiment were retested 3 years later and then tested again after another 2 years. These persons included two alternating suppressors (one esotrope and one exotrope) and two constant suppressors (one esotrope and one exotrope). Variance of markings produced by each subject did not change significantly over the three tests (df $=88,88, F<.7$ or $F>1.4$ ). For the alternating suppressors in this group, IDC 


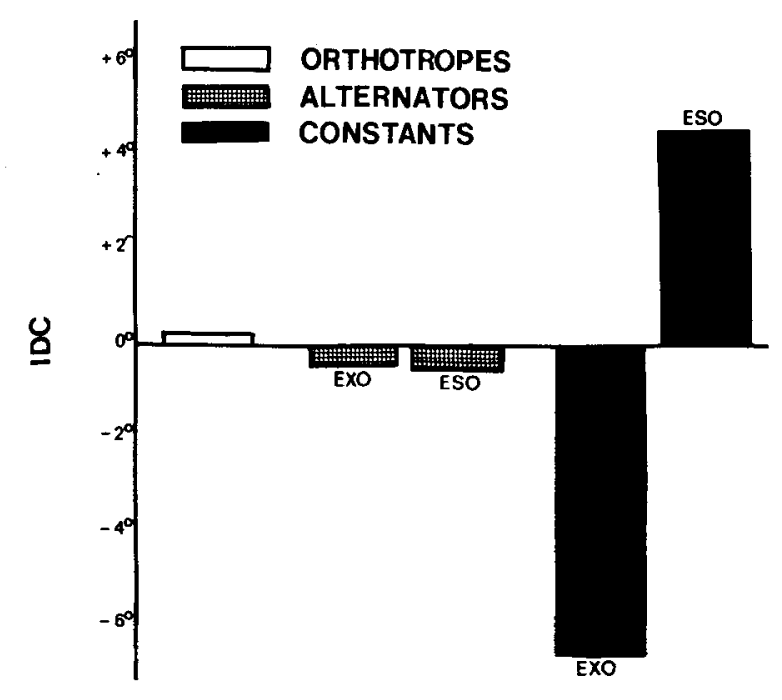

Figure 1. Mean values of IDC in strabismic and nonstrabismic subjects.

was not significantly different from zero in all three tests (df $=22,|t|>1.7)$. For constant suppressors, values of IDC did not significantly increase or decrease over this period $(\mathrm{df}=\mathbf{8},|\mathrm{t}|>1.8)$.

\section{Discussion}

Orthotropes, constant suppressors, and alternating suppressors did not differ in variance of target markings. Nor did markings mediated by the dominant and nondominant eye differ in variance in any group. The latter result differs from that thought by Porac and Coren (1976) to be characteristic of tasks such as batting a baseball (Adams, 1965) and free-throw shooting (Shick, 1971), in which subjects were more accurate using their dominant eyes.

Variance in our task was also unrelated to visual acuity. It seems likely that if overall accuracy had been greater, some of these comparisons might have yielded significant differences. For our present purpose, the uniformity of variance makes clearer the essential effect: among orthotropes and strabismic alternating suppressors, the centroids of markings mediated by each eye do not differ while constant suppressors show a significant and systematic interocular discrepancy. For esotropic constant suppressors, markings mediated by the normally suppressed eye were displaced toward that eye; for exotropic constant suppressors, markings mediated by the normally suppressed eye were displaced away from that eye. This pattern suggests that reaches toward targets viewed by the normally suppressed eye were being affected by the posture of the (occluded) dominant eye.

When a strabismic subject fixates a target with one eye, the fellow eye is directed either toward (esotropia) or away from (exotropia) the side of the viewing eye.
The deviation corresponds to the angle of vergence error. If, in constant suppressors, the momentary position of the dominant eye influences the direction of reaches to targets presented to the normally suppressed eye, the centroids of markings mediated by the two eyes should be separated by a distance positively correlated with the magnitude of vergence error. We examined this possibility in Experiment 2.

For alternating suppressors, the centroids of markings mediated by the two eyes did not differ. This suggests that position of the dominant eye does not influence localization of targets presented to the nondominant eye in all strabismic individuals. In particular, the difference between alternating and constant suppressors in this respect suggests that it is consistent suppression of the nondominant eye which makes position of the dominant eye determinant. Thus, constant suppressors for whom a prolonged period of occlusion of the dominant eye had been instituted as a therapeutic procedure might be expected to behave as do alternating suppressors. A group of subjects who had undergone occlusion therapy prior to age 6 was included in the population studied in Experiment 2 to permit examination of this possibility.

\section{EXPERIMENT 2}

\section{Subjects \\ Method}

Patients seen over a 2-year period by members of the staff of the pediatric clinic of the New England College of Optometry served as the source of subjects. Children for whom parental permission was given and adults who volunteered to participate constituted the pool from which our subjects were selected. All subjects were between the ages of 6 and 24; information about vergence error, pattern of use, visual acuity, and clinical history was provided by members of the clinical staff.

The main subject population comprised 52 individuals who presented a comitant vergence error. In all cases, an error of vergence had been observed prior to age 4 and no attempt at surgical correction or occlusion therapy had been made. All subjects were capable of central fixation (as determined by retinoscopy), and none displayed abnormal retinal correspondence (as determined by tests of afterimage localization and by examination with a haploscope). The group of constant suppressors included 16 esotropes and 9 exotropes; the range of interocular differences in acuity for these individuals was from 4 to 18 Snellen points. The alternating suppressors included 11 esotropes and 16 exotropes; the range of interocular differences in acuity for these subjects was from 0 to 10 Snellen points. Constant and alternating suppressors were comparable in magnitude of vergence error (see Figure 2).

An additional group of eight constant suppressors who had undergone dominant eye occlusion therapy prior to age 6 was tested. These patients were all esotropes. We also tested three nonstrabismic amblyopes in whom vision in the nondominant eye was constantly suppressed. Each of these persons displayed a marked anisometropia. Data from both of these groups were analyzed separately from those of the main population.

\section{Procedure}

The apparatus and procedure were those used in Experiment 1. 


\section{Results \\ Strabismic Subjects (Main Population)}

Variability of markings. For each subject, the variance of markings was computed separately for each target as viewed by each eye. The four target positions did not differ in variance, and interocular differences in variance were also nonsignificant (for both, df $=11,11 ; \mathrm{F}<.3$ or $\mathrm{F}>3.0$ ). Therefore, a pooled variance was computed for each subject, collapsing across targets and eyes. The mean value of $s_{p}$ was $2.35^{\circ}$ with intersubject differences not significantly different from zero $(\mathrm{df}=88,88 ; \mathrm{F}<.7$ or $F>1.4$ ).

Location of markings. For each subject, the centroid of markings mediated by each eye was calculated for each target. Interocular differences in centroids were then computed (right eye minus left eye). For alternating suppressors, as in Experiment 1 , these differences were not significantly different from zero ( $\mathrm{df}=22,|\mathrm{t}|>1.7)$. Values among constant suppressors with vergence errors greater than $4^{\circ}$ were significantly different from zero. Averaging across targets, a mean interocular discrepancy (IDC) was calculated for each subject; these values are plotted against vergence error in Figure 2. For all esotropic constant suppressors, IDC was positive; for all exotropic constant suppressors, IDC was negative. The relation between IDC and magnitude of vergence error for constant suppressors was examined by computing a Spearman rank order correlation. As expected, magnitude of vergence

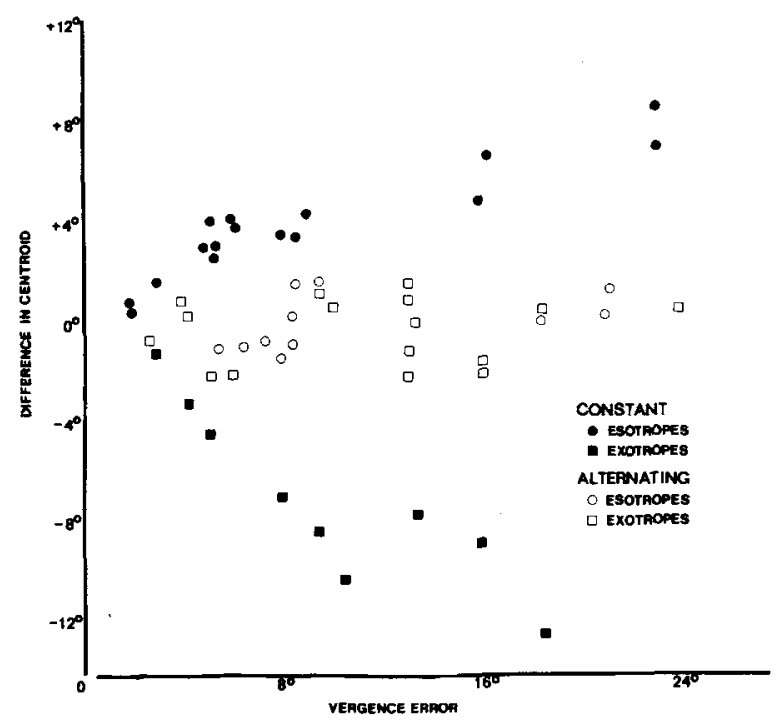

Figure 2. The relation between vergence error and IDC among constant and alternating suppressors. Filled squares $(\boldsymbol{G})$ represent exotropic constant suppressors, filled circles ( $\bullet$ ) represent esotropic constant suppressors; open squares ( $\square$ ) represent exotropic alternating suppressors, open circles $(O)$ represent esotropic alternating suppressors. error was positively correlated with absolute value of IDC among both esotropes $(\mathrm{df}=16, \mathrm{r}=.92$, $\mathrm{p}<.005)$ and exotropes ( $\mathrm{df}=9, \mathrm{r}=.94, \mathrm{p}<.005)$. For both types of misalignment, the absolute value of IDC is equal to approximately $70 \%$ of the vergence error when the degree of vergence error is moderate. As vergence error increases further, the slope of the function appears to decrease.

\section{Other Subject Groups}

Two other groups of subjects were tested and their data analyzed using the methods described above. These additional subjects comprised three nonstrabismic anisometropic amblyopes and eight esotropic constant suppressors whose dominant eye had been patched for an extended period prior to age 6. In agreement with the data from the main group of subjects, intertarget and interocular differences in variance were not significant. For both of the present groups, values of IDC were not significantly different from zero $(\mathrm{df}=22,|\mathrm{t}|>1.7)$. Thus, in the case of nonstrabismic amblyopes, constant suppression of an eye was not associated with an interocular discrepancy in target markings.

The eight constant suppressors who had undergone early occlusion of the dominant eye were all esotropes. In Experiment 1 and in the main population studied in the present experiment, esotropic constant suppressors who had not undergone this therapy had consistently positive values of IDC. In contrast, those constant suppressors who had undergone patching resembled alternating suppressors for whom sign of IDC was not related to type of vergence error.

\section{Discussion}

For constant suppressors in Experiment 1 there was a significant discrepancy in the location of markings mediated by the two eyes. The relative position of markings corresponded to type of misalignment (esotropic vs. exotropic). This relationship suggested that for constant suppressors, the direction of reaches when targets are presented to the normally suppressed eye is influenced by the orbital posture of the (occluded) dominant eye. This hypothesis led us to predict that magnitude of vergence error should correlate with absolute value of IDC. In addition, the sign of IDC was expected to be opposite in esotropes and exotropes. The results of Experiment 2 confirmed both these expectations.

In Experiment 1, alternating suppressors, unlike constant suppressors, showed no interocular discrepancy. Experiment 2 replicated this observation in a second group of strabismic alternators. In addition, a group of constant suppressors in whom the dominant eye had been intermittently occluded prior to age 6 were observed to behave as did alternating 
suppressors. It appears that enforced use of the nondominant eye, while it did not prevent constant suppression of that eye, did prevent reliance on the posture of the dominant eye in localizing targets viewed by the suppressed eye. How this dissociation is to be explained awaits further work which might also specify the period of development during which the preventive effect could be produced and the critical duration of exposure.

In Experiment 1, reduced acuity was observed to have no demonstrable effect on variance in target localization. This was replicated for the strabismic subjects of Experiment 2. In addition, a small number of nonstrabismic amblyopes was observed. Again, there were no significant differences in the variance of markings mediated by each eye. Like strabismic constant suppressors, nonstrabismic amblyopes consistently suppress vision in the nondominant eye. Like other orthotropes, however, they show no interocular discrepancy in the direction of visually guided reaches. This result is consistent with the view that the value of IDC reveals the influence of dominant eye posture on localization of targets viewed by the nondominant eye. IDC is significant, however, only when the two eyes are misaligned.

\section{GENERAL DISCUSSION}

Strabismics in whom vision in one eye is constantly suppressed and strabismics in whom each eye is alternately suppressed differed in the way in which visual targets were localized. Like nonstrabismic individuals, strabismic alternating suppressors localized targets equivalently using either eye. In contrast, strabismic constant suppressors showed a systematic discrepancy in the location of markings mediated by the two eyes. The direction and amount of interocular discrepancy was related to type of vergence error and its extent. To account for this relation, a model was proposed in which the direction of guided reaches to targets viewed by either eye of constant suppressors reflects the momentary orbital position of the dominant eye. When a vergence error is present, this dependency causes markings mediated by the normally suppressed eye to be displaced away from the target.

Inaccurate reaching movements have also been reported in adults who have suffered trauma producing paresis of one or more extraocular muscles. When asked to point toward targets viewed by the damaged eye, they "past point" in a direction determined by the location of the injured muscle. This error results in an interocular discrepancy in centroids which appears similar to what we observed among constant suppressors (von Noorden, Awaya, \& Romano, 1971). However, "past pointing" is a transient phenomenon occurring only at the onset of paretic strabismus (Burian \& von Noorden, 1974), whereas the differences we observed appeared to be stable over a 5-year period and were not associated with paralysis. Moreover, significant values of IDC, while found among constant suppressors, were absent among alternating suppressors, indicating that misalignment of the visual axes is not sufficient to produce this effect. The behavior of constant suppressors when targets are presented to the normally suppressed eye implies that retinal locus information from that eye is combined with postural information from the (occluded) dominant eye. Why should reaches to targets presented to the normally suppressed eye be influenced by the posture of the other eye? The general relation between eye position and localizing behavior is a relevant consideration. The image of a stationary object falls upon a different retinal locus, depending upon the momentary posture of the eye in its orbit. There exists a correspondence between the direction of objects in space, the retinal loci of their images, and the set of orbital postures that the eye can assume. During early devlopment, this correspondence is incorporated in a representation of visual space which supports visually guided behavior. Subsequently, information about retinal locus of an object's image and information about the posture of the eye combine to permit movements to be directed toward that object. For individuals in whom vision in one eye has been constantly suppressed, the relation between posture of that eye, object direction, and retinal locus might not have been extracted. With respect to the dominant eye, this information would have been extracted in the course of using that eye and incorporated in a representation of visual space. This developmental history would leave only information about the posture of the dominant eye to disambiguate retinal locus information when targets are presented to the normally suppressed eye. The source of eye posture information might be either afference from the extraocular muscles of the dominant eye or efference to those muscles. In the absence of such information, the variability of markings of targets presented to the normally suppressed eye would be expected to increase.

For orthotropic subjects in whom vision in both eyes is normally combined, there is evidence that the posture of both eyes influences both binocular and monocular judgments of visual direction (Ono, Wilkinson, Muter, \& Mitson, 1972). In these subjects, information about the posture of both eyes presumably was related to the retinal loci of images during the time when a representation of visual space was acquired. Constant monocular suppression during this period might eliminate monitoring of afference from the muscles of the suppressed eye or monitoring of efference to those muscles. Alternately, 
constant suppression might prevent the afferent or efferent signal from being correlated with information about the retinal locus of images for that eye. In either case, a representation of visual space would be formed based only upon information related to the dominant eye. When constant suppressors are subsequently required to use the normally suppressed eye to mediate guided reaching, they perforce utilize information specifying the posture of the dominant eye, combining it with retinal locus information from the eye of view. For constant suppressors who are strabismic, the fact that the dominant eye is misaligned with the viewing eye results in an interocular discrepancy in target localization. Strabismic alternating suppressors, on the other hand, display no interocular discrepancies in target localization. For them, information about the locus of an image on the retina of the nondominant eye appears to be combined with information about the posture of that eye. This suggests that they have acquired two separate representations of visual space, each of which incorporates the correspondence between object direction, eye posture, and retinal locus for one eye.

A series of studies in kittens has demonstrated that if the animal is provided alternating monocular exposure in light, each eye may independently acquire the capacity to mediate visually guided behaviors (Hein \& Diamond, 1971, 1972). In that case, behaviors mediated by one eye may not be mediated by the other. This suggested a means to confirm the status of the representation of visual space in strabismic alternating suppressors (who, in target localization, are indistinguishable from orthotropes). We have used the pattern of interocular transfer of adaptation to optical transformations of input to provide evidence for the distinctions made here (Mann, Hein, \& Diamond, 1979). That study supports the view that strabismic alternating suppressors utilize two independent monocular representations of visual space, that constant suppressors utilize a representation related to the dominant eye alone and that persons with normal binocularity of vision utilize a single representation of space common to both eyes.

\section{REFERENCES}

ADAms, G. L. Effect of eye dominance on baseball batting. Research Quarterly, 1965, 36, 3-9.
Bauer, I. A., Wood, G. D., \& Held, R. A device for rapid recording of positional responses in two dimensions. Behavior Research Methods \& Instrumentation, 1969, 4, 157-159.

Brenner, R. L., Charles, S. T., \& Flynn, J. T. Pupillary responses in rivalry and amblyopia. Archives of Opthalmology, $1969,82,23-29$.

Burian, H. M., \& von Noorden, G. K. Binocular vision and ocular motility. St. Lou is, Mo: Mosby, 1974.

Franscetti, A. T., \& Burian, H. M. Visually evoked responses in alternating strabismus. American Journal of Ophthalmology 1971, 71, 1292-1297.

Hein, A., \& Diamond, R. M. Contrasting development of visually triggered and guided movements with respect to interocular and interlimb equivalence. Journal of Comparative and Physiological Psychology, 1971, 76, 219-224.

Hein, A., \& Diamond, R. Locomotory space as a pre-requisite for acquiring visually-guided reaching in kittens. Journal of Comparative and Physiological Psychology, 1972, 81, 394-398.

Hein, A., \& Held, R. A neural model for labile sensorimotor coordinations. In E. E. Bernard \& M. R. Kare (Eds.), Biological prototypes and synthetics systems. 1. New York: Plenum Press, 1962. Pp. 71-74.

Held, R., \& Gottlieb, N. Technique for studying adaptation to disarranged hand-eye coordination. Perceptual and Motor Skills, $1958,8,83-86$.

Held, R., \& Hein, A. Movement-produced stimulation in the development of visually-guided behavior. Journal of Comparative and Physiological Psychology, 1963, 56, 872-876.

LEHMULKE, S. W., \& Fox, R. Effects of binocular rivalry summation on the motion after-effect. Vision Research, 1975, 15, 855-858.

Lombrusco, C. T., Duffy, F. H., \& Robr, R. M. Selective suppression of cerebral evoked potential to patterned light in amblyopia ex anopsia. Electrocenphalographic \& Clinical Neurology, 1969, 27, 238-247.

Mann, V., Hein, A., \& Diamond, R. Patterns of interocular transfer of visuomotor coordination reveal differences in the representation of visual space. Perception \& Psychophysics, 1979, 25, 35-41.

Ono, H., Wilkinson, A., Muter, P., \& Mitson, L. Apparent movement and change in perceived location of a stimulus produced by a change in accommodative vergence. Perception \& Psychophysics, 1972, 12. 187-192.

Porac, C., \& Coren, S. The dominant eye. Psychological Bulletin, 1976, 5, 880-897.

Reinecke, R. D., \& Miller, D. Strabismus. New York: Appleton-Century-Croft, 1966.

RIESEN, A. H. Plasticity of behavior. In H. Harlow \& C. Woolsey (Eds.), Biological and biochemical bases of behavior. Madison: University of Wisconsin Press, 1958. Pp. 425-450.

Sнick, I. Relationship between depth perception and hand-eye dominance and free-throw shooting in college women. Perceptual and Motor Skills, 1971, 33, 539-542.

von Noorden, G. K., Awaya, S., \& Romano, P. Past pointing in strabismus. American Journal of Ophthalmology, 1971, 71, 27.33 .

(Received for publication May 2, 1978; revision accepted September 29, 1978.) 